\title{
Optical Second Harmonic Generation and Its Photoinduced Dynamics in Ferroelectric Semiconductor $\mathrm{Sn}_{2} \mathbf{P}_{2} \mathbf{S}_{6}$
}

\author{
K. A. Brekhov ${ }^{a, *}$, K. A. Grishunin ${ }^{a}$, D. V. Afanas'ev ${ }^{b}$, S. V. Semin ${ }^{a, b}$, \\ N. E. Sherstyuk ${ }^{a}$, E. D. Mishina ${ }^{a}$, and A. V. Kimel ${ }^{a, b}$ \\ ${ }^{a}$ Moscow Technological University (MIREA), Moscow, 119454 Russia \\ ${ }^{b}$ Radboud University, Institute for Molecules and Materials, Nijmegen, 6500 GL Netherlands \\ *e-mail: brekhov_ka@mail.ru \\ Received May 31, 2017
}

\begin{abstract}
By means of optical pump-probe technique, the ultrafast dynamics of nonlinear optical response of the ferroelectric semiconductor $\mathrm{Sn}_{2} \mathrm{P}_{2} \mathrm{~S}_{6}$ crystal excited with a femtosecond laser pulse has been investigated. It has been shown that, under the action of femtosecond pulses, change in optical second harmonic generation occurs in the sample, which can be due to screening of existing electric polarization.
\end{abstract}

DOI: $10.1134 / \mathrm{S} 1063783418010080$

\section{INTRODUCTION}

A long march has been made on the way to complete optical switching of magnetization in magnetically ordered media. The first investigations on this subject matter showed that excitation with a laser pulse with duration of $60 \mathrm{fs}$ destroys the magnetic order of a ferromagnetic nickel film on a time scale on the order of $1 \mathrm{ps}$ [1]. After that, the possibility of light excitation of spin oscillations at the magnetic-resonance frequency was demonstrated [2-4]. Although initially the amplitudes of these oscillations did not exceed a few degrees, it has been demonstrated later on that, in the vicinity of a phase transition, these oscillations can be enhanced noticeably [5]. The authors of [6] and [7] demonstrated the possibility of reorientation of spins under the action of a femtosecond laser pulse by $90^{\circ}$ and $180^{\circ}$, correspondingly. Allowing for these results, the possibility of optical data recording using a short laser pulse in an ultimately short time was revealed [8]. Lastly, in works [9-11], the possibility of data recording by means of the action of laser pulses on a magnetic carrier was reported.

It is significant that, despite the obvious analogies between magnetically ordered and ferroelectric materials, the possibility of the ultrafast switching of the order parameter in ferroelectrics has hardly been studied and remains a topical issue, the solution to which will make it possible to create new more efficient ferroelectric memory devices [12].

Earlier [13], we demonstrated the possibility of exciting a soft phonon mode in a ferroelectric semiconductor $\mathrm{Sn}_{2} \mathrm{P}_{2} \mathrm{~S}_{6}$ (SPS) crystal under the action of a femtosecond laser pulse. This may be evidence that light can influence the ferroelectric order parameter and, by analogy with magnetic materials, become the first step toward the complete optical switching of ferroelectric polarization Optical Second Harmonic Generation and Its Photoinduced Dynamics.

A common method for detecting a phase transition is the X-ray diffraction technique. However, this technique is rather intricate for the investigation of a phase transition with time resolution [14].

The technique of optical second harmonic generation (SHG) has been one of the main methods for investigation of the properties of ferroelectric crystals, thin films, and nanostructures since long ago [15-19] and, unlike X-ray diffraction, it enables investigation of the dynamics of the ferroelectric order parameter.

In spite of all advantages, at present, there are only several works dealing with application of the SHG method to investigation of such a class of materials as ferroelectric semiconductors [20-23], in which observation of optical switching of the ferroelectric order parameter is most probable due to screening of polarization by charge carriers photoexcited to the conduction band.

In the present work, the excitation and dynamics of the nonlinear optical response of a ferroelectric semiconductor SPS crystal was investigated using a femtosecond laser pulse. It was revealed that, under the action of femtosecond pulses, change in the SHG intensity occurs in the sample, which can be associated with screening of existing electric polarization. 


\section{EXPERIMENTAL SETUP AND TECHNIQUE}

In the investigations, a tin hypothiodiphosphate $\mathrm{Sn}_{2} \mathrm{P}_{2} \mathrm{~S}_{6}$ crystal cut along crystallographic plane [010] was employed.

The SPS is an intriguing crystal from the researcher's point of view, a wide-bandgap semiconducting ferroelectric with unique nonlinear optical properties and a wide optical radiation transmission bandwidth in the range from $530 \mathrm{~nm}$ to $8 \mu \mathrm{m}$. At room temperature, the SPS crystal is a ferroelectric with monoclinic point symmetry group $m$ and undergoes a second-order phase transition into a paraelectric phase with point symmetry group $2 / m$ at a temperature about $337 \mathrm{~K}$ [24]. At the same time, this material behaves as a wide-bandgap semiconductor with a band-gap energy of $E_{g}=2.31 \mathrm{eV}$. The wavelengths for the pump-probe experiment were chosen on the basis of the results given in article [25], in which it was shown that, for the electromagnetic radiation with the energy of photons lower than $2 \mathrm{eV}$, the absorption coefficient in this material is extremely low $\left(\leq 3 \mathrm{~cm}^{-1}\right)$, while, at the energies higher than $2 \mathrm{eV}$, the absorption coefficient increases rapidly reaching the values on the order of $10^{5} \mathrm{~cm}^{-1}$. Such a behavior is typical of semiconducting materials, as well as the photoconductivity phenomenon, which was studied with SPS in [25, 26].

The energy-level diagram of the SPS crystal is given in [25]. It is shown that the conduction band consists of several narrow subbands. In work [27], it was asserted that, owing to $\mathrm{Sn}^{+}$ions, acting as acceptors, undoped SPS is a $p$-type semiconductor at room temperature.

For the investigation of the SHG relaxation kinetics in the SPS crystal, an optical pump-probe technique was used. The scheme of the experimental setup is analogous to that given in work [28]. As a radiation source, pulses generated by a solid-state laser on a sapphire crystal doped with titanium ions were employed. The central radiation wavelength of such a laser was $800 \mathrm{~nm}$. Next, the radiation was passed through an amplifier and compressor so that to eventually obtain a sequence of laser pulses with a duration of each pulse of 80 to $100 \mathrm{fs}$ and a repetition rate of $1 \mathrm{kHz}$. After that, the radiation was split with a beam splitter between the probe radiation and pump radiation channels in the intensity ratio of $3: 7$.

The spectrum of the pump beam could be tuned with the help of an optical parametric amplifier. Pump radiation with a wavelength of $570 \mathrm{~nm}$ (photon energy $2.18 \mathrm{eV}$ ) passed through a delay line was focused onto the sample surface. The pump beam was impinging on the sample parallel to the sample surface normal.

The central wavelength of the probe pulse was chosen to be $800 \mathrm{~nm}$ (photon energy $1.55 \mathrm{eV}$ ). Polarization of both beams was linear. The angle of incidence of the probe beam was $45^{\circ}$. Detection took place at the second harmonic wavelength $(400 \mathrm{~nm})$ with the use of a photoelectric multiplier (PEM), the signal from which was processed with a photons counter (Stanford Research). To detect the signal only at the second harmonic wavelength, other radiation was filtered with a narrow-band filter. It is worthy of note that, even at relatively low intensities of the exciting radiation, the sample surface can be damaged as a result of the laser radiation action. This can be due to a "fatigue" effect, when each exciting pulse creates and leaves microdefects, accumulation of which leads to destruction of the sample. Therefore, the choice of the exciting radiation power is greatly important for the interpretation of the experimental results.

For the sample heating, the experimental setup was equipped with a heating element and a controller that allows one to vary and hold the preset temperature.

\section{OPTICAL SECOND HARMONIC GENERATION \\ 3.1. Spectral Dependence}

To reveal the spectral features of the ferroelectric semiconductor SPS crystal, the spectral dependences of the SHG and luminescence upon two-photon excitation (TPL) were investigated in the wavelength range of $730-950 \mathrm{~nm}$. Figure 1 shows the characteristic luminescence spectra of the SPS crystal at pump wavelengths of $800(1.55 \mathrm{eV}), 840(1.48 \mathrm{eV}), 880(1.41$ $\mathrm{eV})$, and $900 \mathrm{~nm}(1.38 \mathrm{eV})$. The short-wavelengths narrow peaks correspond to generation of radiation at a doubled frequency, and wider peaks centered around $2.25 \mathrm{eV}(550 \mathrm{~nm})$ correspond to luminescence upon two-photon absorption. With an increase in the pump wavelength from 750 to $950 \mathrm{~nm}$, intensity of the SHG peak rises sharply, increasing by approximately two orders of magnitude. The intensity of the luminescence peak is maximum at the pump wavelength in the

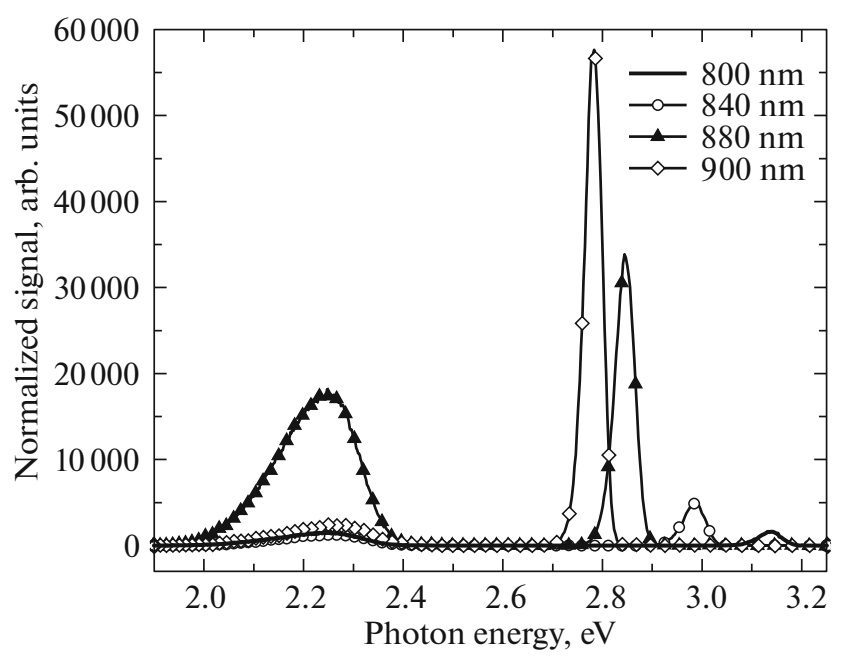

Fig. 1. Luminescence spectra of the SPS crystal at pump wavelengths of $800,840,880$, and $900 \mathrm{~nm}$. 
vicinity of $880 \mathrm{~nm}$, and its position shifts slightly (within $10 \mathrm{~nm}$ ) toward shorter wavelengths with an increasing pump wavelength.

\subsection{Second Harmonic Generation Temperature Dependence}

Phenomenologically, the process of second optical-harmonic generation is described using nonlinear polarization. Polarization induced by a light field is described by the constitutive equation

$$
\mathbf{P}=\mathbf{P}(\mathbf{E}) \text {, }
$$

which reflects the structure and properties of the medium. The simplest constitutive equation of a nonlinear medium has the form

$$
P=\chi^{(1)} E+\chi^{(2)} E^{2}+\chi^{(3)} E^{3}+\ldots+\chi^{(m)} E^{m}+\ldots
$$

According to this equation, polarization of a medium is a nonlinear function of the light field strength $E$.

Separating linear and nonlinear components in the polarization of the medium, one can write

$$
\mathbf{P}=\mathbf{P}_{l}+\mathbf{P}_{n l},
$$

where

$$
\begin{gathered}
P_{l}(E)=\chi^{(1)} E, \\
P_{n l}(E)=\chi^{(2)} E^{2}+\chi^{(3)} E^{3}+\ldots+\chi^{(m)} E^{m}+\ldots
\end{gathered}
$$

Here $\chi^{(m)}$ is nonlinear susceptibilities of the respective order. The coefficients $\chi^{(m)}$ held by the terms of expansion (5) are tensors of the order $(m+1)$. Then, the wave equation obtained from the Maxwell equations will take the form

$$
\operatorname{curl} \operatorname{curl} \mathbf{E}+\frac{1}{c^{2}} \frac{\partial^{2} \mathbf{E}}{\partial t^{2}}+\frac{4 \pi}{c^{2}} \frac{\partial^{2} \mathbf{P}_{l}}{\partial t^{2}}=-\frac{4 \pi}{c^{2}} \frac{\partial^{2} \mathbf{P}_{n l}}{\partial t^{2}}
$$

for a nonlinear anisotropic medium and

$$
\Delta \mathbf{E}-\frac{1}{c^{2}} \frac{\partial^{2} \mathbf{E}}{\partial t^{2}}-\frac{4 \pi}{c^{2}} \frac{\partial^{2} \mathbf{P}_{l}}{\partial t^{2}}=\frac{4 \pi}{c^{2}} \frac{\partial^{2} \mathbf{P}_{n l}}{\partial t^{2}}
$$

for a nonlinear isotropic medium.

These equations show that nonlinear polarization of the medium is the source of new spectral components of the field [29].

The term of expansion (5) that depends on the light-wave field strength quadratically will be the source of radiation at a doubled frequency. Let in a medium with a nonlinear susceptibility of the second order $\chi^{(2)}$ propagate a monochromatic plane light wave

$$
E(r, t)=E_{0} \exp \{-i \omega t+k r\} .
$$

Upon propagation of the wave in a nonlinear medium, a polarization wave

$$
P_{n l}(2 \omega)=\chi^{(2)} E_{0}^{2} \exp \{-i 2 \omega t+2 k r\}
$$

will be excited.
This wave at a radiation frequency of $2 \omega$ will be the wave that is the source of second harmonic radiation.

An important advantage of the SHG technique is the sensitivity to change in the crystal structure of the sample during a phase transition. In particular, upon transition from a noncentrosymmetric to a centrosymmetric phase, the intensity of second opticalharmonic generation decreases by many times. This is caused by the fact that, for this type of symmetry, SHG in an electrodipole approximation, which is written as

$$
\mathbf{P}^{D}(2 \omega)=\hat{\chi}^{D(2)} \mathbf{E}(\omega) \mathbf{E}(\omega)
$$

is prohibited, since, in a centrosymmetric medium, $\hat{\chi}^{D(2)}=0$.

For a centrosymmetric medium, the mechanism of nonlinear polarization generation is quadrupole and is written as

$$
\mathbf{P}^{Q}(2 \omega)=\hat{\chi}^{Q(2)} \mathbf{E}(\omega) \nabla \mathbf{E}(\omega) ;
$$

therefore, the intensity of SHG decreases by 6 to 8 orders of magnitude [30, 31].

In ferroelectric crystals with a centrosymmetric high-temperature phase, the components of the tensor of nonlinear susceptibility $\hat{\chi}$ are proportional to ferroelectric polarization [32]. At the same time, since the intensity of the second harmonic $(\mathrm{SH}) I_{2 \omega}$ is proportional to the squared field strength of the $\mathrm{SH}$

$$
E_{2 \omega} \propto E^{2}(2 \omega),
$$

we obtain the relationship between the intensity of the $\mathrm{SG}$ and ferroelectric polarization in the form

$$
I_{2 \omega} \propto\left(P^{0}\right)^{2} .
$$

Figure 2 shows the SHG temperature dependence of the SPS crystal. It is seen that, with an increasing temperature, the intensity of harmonic generation goes down rapidly, approaching zero at temperatures higher than the critical one. This is evidence that the crystal structure passes from a ferroelectric phase to a paraelectric phase, in which $\mathrm{SHG}$ is prohibited.

According to Eq. (13), the intensity of the $\mathrm{SH}$ is proportional to the squared polarization, which is the order parameter in ferroelectrics.

In the context of the Landau theory, in the mean field model, the temperature dependence of the order parameter is described by the expression [33]

$$
P \propto\left(T_{c}-T\right)^{1 / 2} .
$$

Therefore,

$$
I_{2 w} \propto\left(T_{c}-T\right) .
$$

It is seen that the dependence in Fig. 2 has two pronounced linear pieces. Fitting this dependence by two straight lines and finding the place where they intersect, one can determine the Curie temperature of $336 \mathrm{~K}$, which agrees well with the literature. 


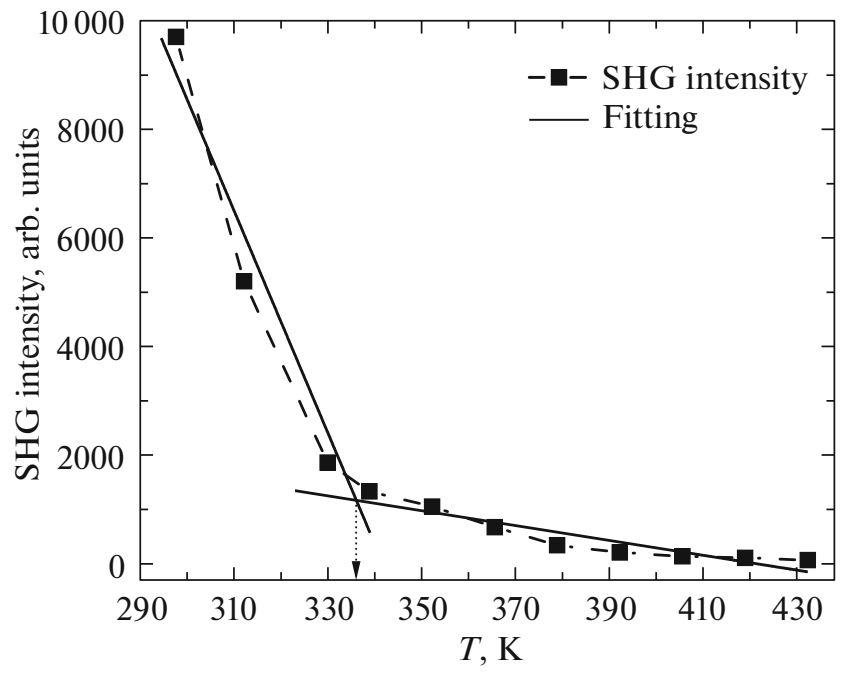

Fig. 2. The SHG intensity dependence on sample heating in the SPS crystal.

The nonzero SH signal immediately after the Curie point can be explained by "smearing" of the phase transition due to the presence of defects in the nearsurface layer and at the crystal surface. In these regions, weak SHG is observed even at temperatures higher than the Curie temperature as a result of disturbance of central symmetry of the structure.

\section{PHOTOINDUCED DYNAMICS OF OPTICAL SECOND HARMONIC GENERATION}

\subsection{The Nonlinear Optical Response Relaxation Kinetics}

The light-induced dynamics of excitation and relaxation of the intensity of SHG at room temperature were investigated at time delays between the excitation and probe pulses up to $2.5 \mathrm{~ns}$.

The results of this experiment are illustrated in Fig. 3 . It is seen that excitation of a ferroelectric semiconductor with photons with an energy of $2.18 \mathrm{eV}$ leads to a dramatic change in the intensity of SHG. The changes occur on a time scale on the order of the pulse duration. Then, the photoinduced signal relaxes exponentially. The experimental data were fitted with a function based on the approach developed earlier for the description of similar processes in direct-gap semiconductors [34]:

$$
\begin{aligned}
& y=A_{\exp }\left(\frac{w^{2}}{\tau_{1}^{2}}-\frac{\tau_{d}}{\tau_{1}}\right)\left[1-\operatorname{erf}\left(\frac{w}{\tau_{1}}-\frac{\tau_{d}}{2 w}\right)\right] \\
& +B_{\exp }\left(\frac{w^{2}}{\tau_{2}^{2}}-\frac{\tau_{d}}{\tau_{1}}\right)\left[1-\operatorname{erf}\left(\frac{w}{\tau_{2}}-\frac{\tau_{d}}{2 w}\right)\right],
\end{aligned}
$$

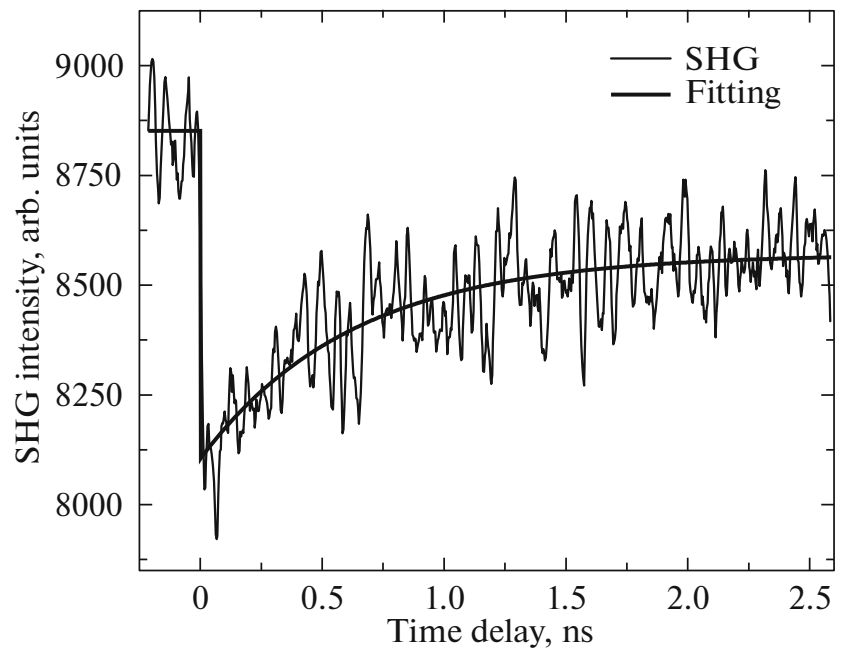

Fig. 3. The SHG intensity dependence on time delay between excitation and probe pulses at room temperature.

where $\tau_{d}$ is the time delay between excitation and probe pulses, $\tau_{1}$ and $\tau_{2}$ are the times of thermalization and recombination of charge carriers, respectively, $A$ and $B$ are constants, and $w$ is the pulse duration. We established earlier that the thermalization time in this material is $\tau_{1}=1.62 \pm 0.03$ ps [13]. Taking that into account, in this work, we found that the characteristic time of recombination of electrons from the conduction band to the valence band is $\tau_{2} \sim 620 \mathrm{ps}$.

\subsection{The Temperature Dependence of Nonlinear Optical Response Relaxation Kinetics}

To perceive the nature of photoinduced changes in the nonlinear optical response, the dynamics of second optical-harmonic generation were investigated in the time range up to $5 \mathrm{ps}$ at various temperatures. The experimental results are given in Fig. 4. It is seen from the figure, that, when the sample temperature is increased, the value $\Delta I$ changes, which is the relative change in the signal of the intensity of SHG determined as the height of a "step" in the SHG signal between the regions $\tau_{d}<0$ and characteristic times of thermalization of carriers. Also of notice is the change in the character of the time dependence in the region up to 1 ps at temperatures higher than the critical one. This issue demands further investigation and is not considered in the present study.

Figure 5 shows the dependence of $\Delta I$ on sample heating temperature. The size of symbols in the figure is greater than the error in each point.

The change in $\Delta I$ is proportional to a change in squared ferroelectric polarization: $\Delta I \propto \Delta P^{2}$. Upon heating,

$$
\Delta P=\frac{d P}{d T} \Delta T
$$




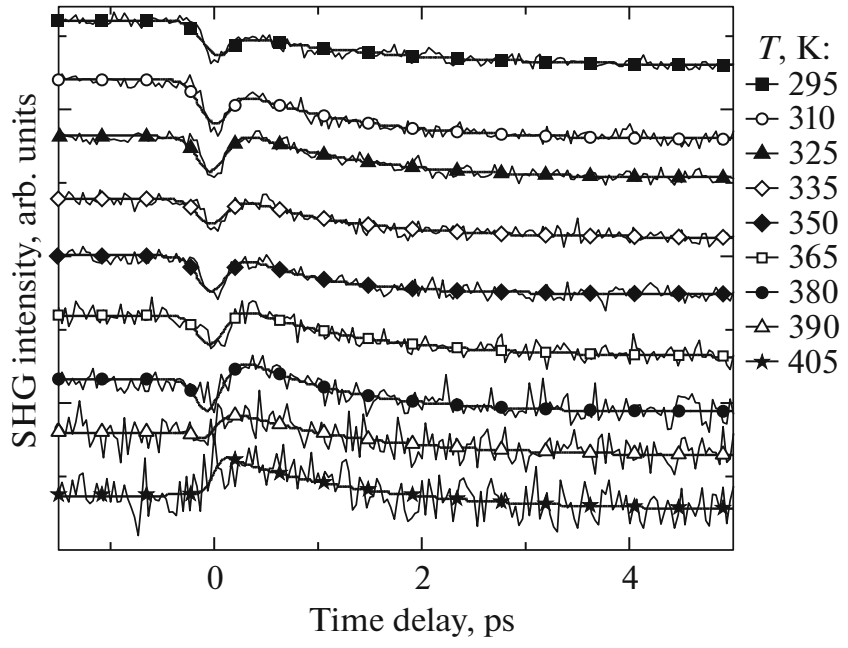

Fig. 4. The SHG intensity dependence on $\tau_{d}$, which is the time delay between excitation and probe pulses at various temperatures. The SG signal is normalized to generation intensity in the range $\tau_{d}<0$. Solid lines denote the experimental data, lines with symbols denote the fitting.

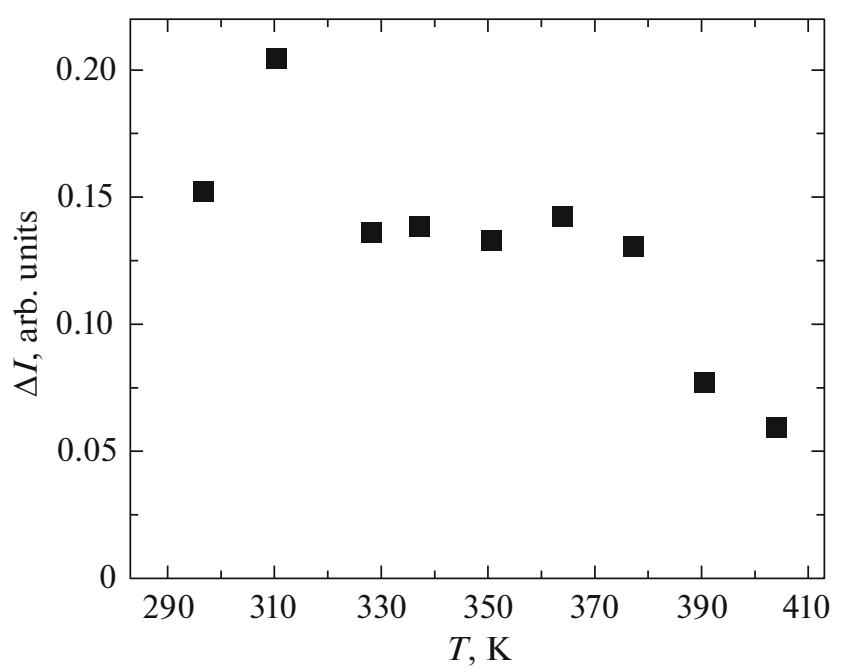

Fig. 5. Dependence of $\Delta I$ on sample temperature.

which means that

$$
\Delta I \propto \frac{d P^{2}}{d T} \Delta T .
$$

$\Delta T$ is change in temperature and does not depend on the actual sample temperature. $P^{2} \propto\left(T_{c}-T\right)$; hence $d P^{2} / d T$ does not depend on the sample temperature either. Thus we obtain that $\Delta I$ is also not a function of temperature.

It is seen from Fig. 2 that, upon heating, $d P^{2} / d T$ changes in the Curie point.

Therefore, comparing the dependences in Figs. 2 and 5 in the region of the phase transition, one can draw a conclusion that the observed dynamics of the intensity of the SHG signal is not connected with ultrafast laser heating of a crystal or with an effective change in the phase transition point, since it is equivalent to heating/cooling of the sample relative to the Curie point. The most probable reason for the observed photoinduced changes is screening by photoinduced charge carriers of that part of electric polarization which is probed by the second optical harmonic. In this process, not all photoexcited charge carriers are relaxed. A portion of them is captured by long-lived states and creates defects, accumulation of which leads to destruction of the sample.

\section{CONCLUSIONS}

To conclude, spectral dependences of SHG and luminescence upon two-photon excitation were investigated for the ferroelectric semiconductor crystal $\mathrm{Sn}_{2} \mathrm{P}_{2} \mathrm{~S}_{6}$. It was established that, with an increasing wavelength of the exciting radiation from 750 to $950 \mathrm{~nm}$, the intensity of SHG rises sharply, increasing by approximately two orders of magnitude, and the intensity of the luminescence peak is maximum at the pump wavelength in the vicinity of $880 \mathrm{~nm}$.

The investigation of relaxation of the light-induced dynamics of SHG at room temperature and time delays between the excitation and probe pulses up to 2.5 ns made it possible to determine that the characteristic time of recombination of electrons from the conduction band to the valence band is $\tau_{2} \sim 620 \mathrm{ps}$.

On the basis of the character of the time dependences of the intensity of SHG measured at various temperatures, a conclusion is drawn that the action of light does not lead to ultrafast heating or change in the Curie point, and the most probable reason for the observed changes in the photoinduced dynamics of the intensity of SHG is screening by photoinduced charge carriers of that part of electric polarization, which is probed by the second optical harmonic.

\section{ACKNOWLEDGMENTS}

The work was carried out under the financial support of the Ministry of Education and Science of the Russian Federation (grant 14.Z50.31.0034, state assignment 3.7500.2017/9.10).

\section{REFERENCES}

1. E. Beaurepaire, J. C. Merle, A. Daunois, and J. Y. Bigot, Phys. Rev. Lett. 76, 4250 (1996).

2. G. Ju, A. Nurmikko, R. Farrow, R. Marks, M. Carey, and B. Gurney, Phys. Rev. Lett. 82, 3705 (1999).

3. M. Van Kampen, C. Jozsa, J. T. Kohlhepp, P. LeClair, L. Lagae, W. J. M. de Jonge, and B. Koopmans, Phys. Rev. Lett. 88, 227201 (2002).

4. A. V. Kimel, A. Kirilyuk, P. A. Usachev, R. V. Pisarev, A. M. Balbashov, and T. Rasing, Nature 435, 655 (2005). 
5. E. G. Galkina, I. Y. Mikhailov, and B. A. Ivanov, JETP Lett. 93, 711 (2011).

6. A. V. Kimel, A. Kirilyuk, A. Tsvetkov, R. V. Pisarev, and T. Rasing, Nature 429, 850 (2004).

7. C. D. Stanciu, F. Hansteen, A. V. Kimel, A. Kirilyuk, A. Tsukamoto, A. Itoh, and T. Rasing, Phys. Rev. Lett. 99, 047601 (2007).

8. K. Vahaplar, A. M. Kalashnikova, A. V. Kimel, D. Hinzke, U. Nowak, R. Chantrell, A. Tsukamoto, A. Itoh, A. Kirilyuk, and T. Rasing, Phys. Rev. Lett. 103, 117201 (2009).

9. S. Mangin, M. Gottwald, C. H. Lambert, D. Steil, V. Uhlír, L. Pang, M. Hehn, S. Alebrand, M. Cinchetti, G. Malinowski, Y. Fainman, M. Aeschlimann, and E. E. Fullerton, Nat. Mater. 13, 286 (2014).

10. C.-H. Lambert, S. Mangin, B. S. D. C. S. Varaprasad, Y. K. Takahashi, M. Hehn, M. Cinchetti, G. Malinowski, K. Hono, Y. Fainman, M. Aeschlimann, and E. E. Fullerton, Science 345, 1337 (2014).

11. A. Stupakiewicz, K. Szerenos, D. Afanasiev, A. Kirilyuk, and A. V. Kimel, Nature 542, 71 (2017).

12. G. A. Garrett, J. F. Whitaker, A. K. Sood, and R. Merlin, Opt. Express 1, 385 (1997).

13. K. A. Brekhov, K. A. Grishunin, D. V. Afanas'ev, S. V. Semin, N. E. Sherstyuk, G. K. Kitaeva, E. D. Mishina, T. Rasing, and A. V. Kimel, JETP Lett. 102, 372 (2015).

14. S. Grübel, J. A. Johnson, P. Beaud, C. Dornes, A. Ferrer, V. Haborets, L. Huber, T. Huber, A. Kohutych, T. Kubacka, M. Kubli, S. O. Mariager, J. Rittmann, J. I. Saari, Y. Vysochanskii, G. Ingold, and S. L. Johnson, arXiv (2016).

15. T. F. Heinz, in Nonlinear Surface Electromagnetic Phenomena, Ed. by H. Ponath and G. Stegeman (NorthHolland, Amsterdam, 1991), p. 353.

16. L. V. Simagina, E. D. Mishina, S. V. Semin, N. A. Ilyin, T. R. Volk, R. V. Gainutdinov, and L. I. Ivleva, J. Appl. Phys. 110, 52015 (2011).

17. E. D. Mishina, Ferroelectrics 314, 57 (2005).

18. E. D. Mishina, N. É. Sherstyuk, V. O. Val'dner, A. V. Mishina, K. A. Vorotilov, V. A. Vasil'ev, A. S. Sigov, M. P. de Santo, E. Cazzanelli, R. Barberi, and T. Rasing, Phys. Solid State 48, 1210 (2006).
19. E. D. Mishina, N. E. Sherstyuk, V. I. Stadnichuk, A. S. Sigov, V. M. Mukhorotov, Y. I. Golovko, A. van Etteger, and T. Rasing, Appl. Phys. Lett. 83, 2402 (2003).

20. A. Anema, A. Grabar, and T. Rasing, Ferroelectrics 183, 181 (1996).

21. K. A. Brekhov, K. A. Grishunin, and O. V. Samotokhin, Fundam. Probl. Radioelektron. Priborostroen. 14, 59 (2014).

22. K. A. Grishunin, K. A. Brekhov, and O. V. Samotokhin, Ross. Tekhnol. Zh. 2 (7), 134 (2015).

23. W. Q. Liao, Y. Zhang, C. L. Hu, J. G. Mao, H.-Y. Ye, P. F. Li, S. D. Huang, and R. G. Xiong, Nat. Commun. 6, 7338 (2015).

24. Y. M. V. R. M. Yevych, Condens. Matter Phys. 11, 417 (2008).

25. R. V. Gamernyk, Y. P. Gnatenko, P. M. Bukivskij, P. A. Skubenko, and A. G. Slivka, J. Phys.: Condens. Matter 18, 5323 (2006).

26. A. A. Grabar, Ferroelectrics 192, 155 (1997).

27. Y. M. Vysochanskii, K. Glukhov, M. Maior, K. Fedyo, A. Kohutych, V. Betsa, I. Pritz, and M. Gurzan, Ferroelectrics 418, 124 (2011).

28. R. Medapalli, I. Razdolski, M. Savoini, A. R. Khorsand, A. Kirilyuk, A. V. Kimel, T. Rasing, A. M. Kalashnikova, A. Tsukamoto, and A. Itoh, Phys. Rev. B 86, 54442 (2012).

29. S. A. Akhmanov and S. Yu. Nikitin, Physical Optics (Nauka, Moscow, 2004) [in Russian].

30. N. Bloembergen, Nonlinear Optics: A Lecture Note and Reprint Volume (W. A. Benjamin, New York, 1965).

31. R. R. Birss, Symmetry and Magnetism (North-Holland, Amsterdam, 1964).

32. G. Dolino, J. Lajzerowicz, and M. Vallade, Phys. Rev. B 2, 2194 (1970).

33. M. E. Lines and A. M. Glass, Principles and Application of Ferroelectric and Related Materials (Clarendon, Oxford, 1977).

34. A. Kimel, F. Bentivegna, V. Gridnev, V. Pavlov, R. Pisarev, and T. Rasing, Phys. Rev. B 63, 1 (2001).

Translated by Z. Smirnova 